\title{
A Health Economic Evaluation of Aspirin in the Primary Prevention of Cardiovascular Disease in Japan
}

\author{
Kiichiro Tsutani ${ }^{1}$, Ataru Igarashi ${ }^{1}$, Keita Fujikawa ${ }^{2}$, Thomas Evers ${ }^{3}$, Maria Kubin ${ }^{3}$, \\ Mark Lamotte ${ }^{4}$ and Lieven Annemans ${ }^{4}$
}

\begin{abstract}
Objective Low-dose aspirin is standard care in patients with a history of cardiovascular disease (CVD). But, the use of low-dose aspirin in primary prevention has not yet been fully established in Japan although meta-analyses and US/European guidelines support its use in persons at increased CVD risk. This study assessed the health economic consequences of the use of low-dose aspirin in the primary prevention of CVD in Japan.

Patients and Methods Based on results reported in two recent meta-analyses of Hayden (2002) and Eidelman (2003), a Markov model was constructed to predict the cost-effectiveness of low-dose aspirin in the primary prevention of CVD. The model consists of 5 health states: 1) no history of CVD, 2) history of stroke, 3) history of myocardial infarction, 4) history of CVD, and 5) death, with a 10-year time horizon and 1-year cycles. Direct costs from the insurers' perspective were used, while health outcome was expressed in LifeYears Gained (LYG). 'Discounting Rate' with 3\% was applied on effectiveness and costs.

Results For patients with a 1-year risk of coronary heart disease (CHD) of $1.5 \%$ (10-year risk of $\pm 15 \%$ ), the model demonstrated 'dominance' of the 'aspirin' arm versus 'no aspirin' arm; the 10-year costs were Japanese Yen (JPY) 634,000 (Euro 4,857) and JPY 518,000 (Euro 3,968) in the 'no aspirin' arm and 'aspirin' arm, respectively, while LYG was 8.33 and 8.36 , respectively. Low-dose aspirin treatment saved on average JPY 116,000 (Euro 889) [95\% confidence interval (CI) JPY 57,077-175,151] per patient. Dominance was demonstrated (non-significant) in the first year of treatment and, low-dose aspirin was dominant to 'no aspirin' arm from an annual risk of $0.20 \%$. Other results of sensitivity analysis on gastrointestinal (GI) bleeding rate, stroke rate, cost of each event and discounting showed the robustness of the results.

Conclusions Administering low-dose aspirin to patients with a 1-year risk of CHD of $1.5 \%$ and more is significantly costsaving from the insurers' perspective in Japan.
\end{abstract}

Key words: aspirin, primary prevention, pharmacoeconomics analysis

(DOI: 10.2169/internalmedicine.46.1843)

\section{Introduction}

It is estimated that about Japanese Yen (JPY) 5 trillion (Euro 38 billion: Euro $1=$ JPY 130) National Health Insurance (NHI) budget has been spent on cardiovascular disease (CVD) (about JPY 690 billion (Euro 5.3 billion) for ischemic heart disease (IHD), about JPY 1.7 trillion (Euro 13 billion) for cerebrovascular disease), according to statistics in 2003 of Ministry of Health, Labour and Welfare
(MHLW) (1). As increase in the elderly population would lead to an increase of CVD; cost for treatment of CVD would further be increased in the current situation, although, in the current health care environment in Japan, the budget is quite strictly limited. Hence, health care interventions are no longer valued on efficacy and safety alone. The costeffectiveness is becoming an increasingly important criterion to assess medical technologies.

As an approach to reduce the incidence of CVD, the importance of primary/secondary prevention of CVD is being

${ }^{1}$ The Department of Pharmacoeconomics, Graduate School of Pharmaceutical Sciences, The University of Tokyo, Tokyo, ${ }^{2}$ Product Development Division, Bayer Yakuhin Ltd., Osaka, ${ }^{3}$ Product Development Division, Bayer Health Care, Wuppertal and ${ }^{4}$ HEDM, Brussel Received for publication March 30, 2006; Accepted for publication October 30, 2006 Correspondence to Dr. Keita Fujikawa, keita.fujikawa.kf@bayer.co.jp 


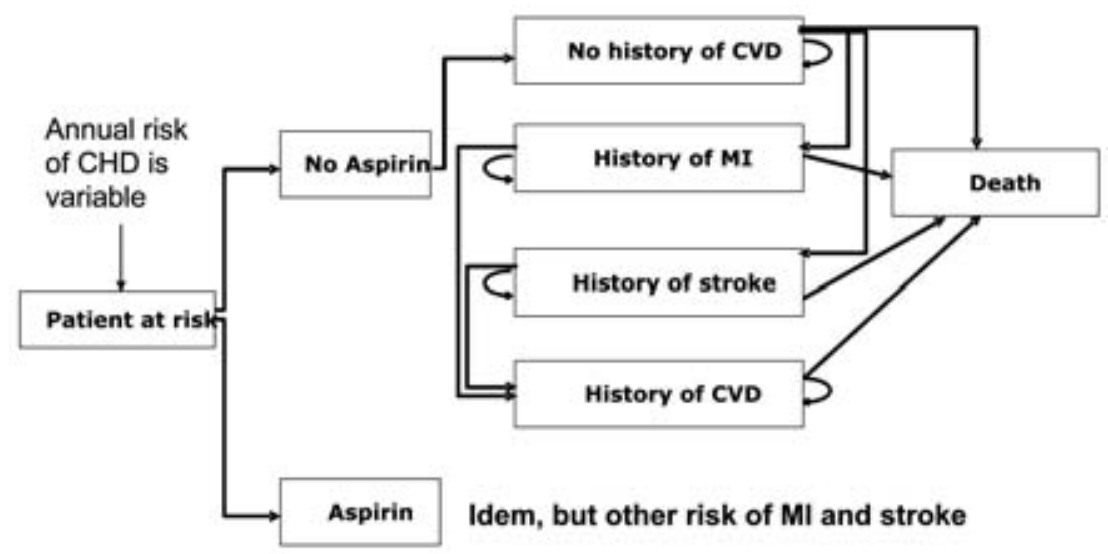

Figure 1. Markov state and transition used in the analysis. CHD: coronary heart disease, CVD: cardiovascular disease, MI: myocardial infarction.

stressed recently. And, low-dose aspirin is currently used for the treatment of patients after having angina, myocardial infarction and TIA, as the golden standard of the secondary prevention of $\operatorname{CVD}(2,3)$.

Furthermore, recently, the importance of primary prevention using aspirin has also been proposed for patients with high CVD risk (without proven atherothrombosis). The results of meta-analyses $(2002,2003)$ support its use in persons at increased CVD risk $(4,5),(2002)$ and the American Heart Association (AHA) recommends to start treatment with aspirin as soon as the risk of a cardiac event is higher than $10 \%$ over a period of ten years (annual risk $\pm 1 \%$ ) (6). In a clinical practice guideline in Europe, the tentative threshold is the same as for statins, a $20 \%$ risk of coronary heart disease (CHD) (annual risk 2\%) (7). And recently, pharmacoeconomics analysis on aspirin use for primary prevention has also been reported in Europe; it demonstrated that aspirin use could save NHI budget (8).

Compared to the clinical evidence and economic analysis available in Europe / North America, the use of low-dose aspirin for primary prevention has not yet been fully established in Japanese population. Therefore, it is also not clear whether or not aspirin use for primary prevention can reduce NHI budget in Japan by reducing the incidence of CVD, despite increasing societal interest.

Hence, this study assessed the health economic consequences of the use of low-dose aspirin in the primary prevention of CVD in Japan, based on a Markov model using meta-analysis data $(4,5)$ and health cost in Japan.

\section{Patients and Methods}

\section{Design}

To predict the cost-effectiveness of low-dose aspirin in the primary prevention of CVD, a Markov model (Figs. 1, 2) was constructed in DATA Pro (release 2, 2002) from TreeAge $^{\mathrm{TM}}$.

(1) Markov state

The following health states were chosen in the Markov model to represent clinically and economically important events in the disease process;

- no history of CVD, meaning no known coronary heart disease, peripheral arterial disease or cerebrovascular disease

- history of stroke

- history of myocardial infarction (MI)

- history of CVD: having both stroke and MI

- death

The analysis includes all harms (eg, hemorrhagic strokes and gastro-intestinal (GI) bleeding) and benefits (eg, less MI and CHD death). The risks depended on whether or not the patient was treated with low-dose aspirin. Hence, the model was run for two possible strategies: 'Aspirin arm' versus 'No aspirin arm'. Results were reported as cost per life year gained (LYG).

(2) Transition from a state to another

(i) Primary prevention

All patients started the model without a history of CVD, then, each year the patient had a risk of CHD defined as (1) a MI or CHD (fatal or not), (2) stroke (fatal or not), or (3) of dying from other causes. Independent of the above risks the patient had a risk of GI bleeding.

Patients that suffered from a fatal event moved to the "death state", whereas patients that underwent a first stroke moved to the state "history of stroke", patients suffering from a first non-fatal myocardial infarction event moved to the state "history of MI"; the remaining patients stayed in the "no history of CVD" state.

(ii) Secondary prevention

Also all patients with a history of stroke or MI again have a risk to suffer from a new event. The patient may have a non-fatal MI or stroke or a systemic bleeding or die from a vascular or other causes. It was assumed that all patients (both treatment groups) were treated with low-dose aspirin after the occurrence of the first event.

(3) Base case

Benefit/risk of aspirin depends on the baseline risk of CHD in the studied population. For the modeling purpose, in base case, patients with a 1-year risk of CHD of $1.5 \%$ 


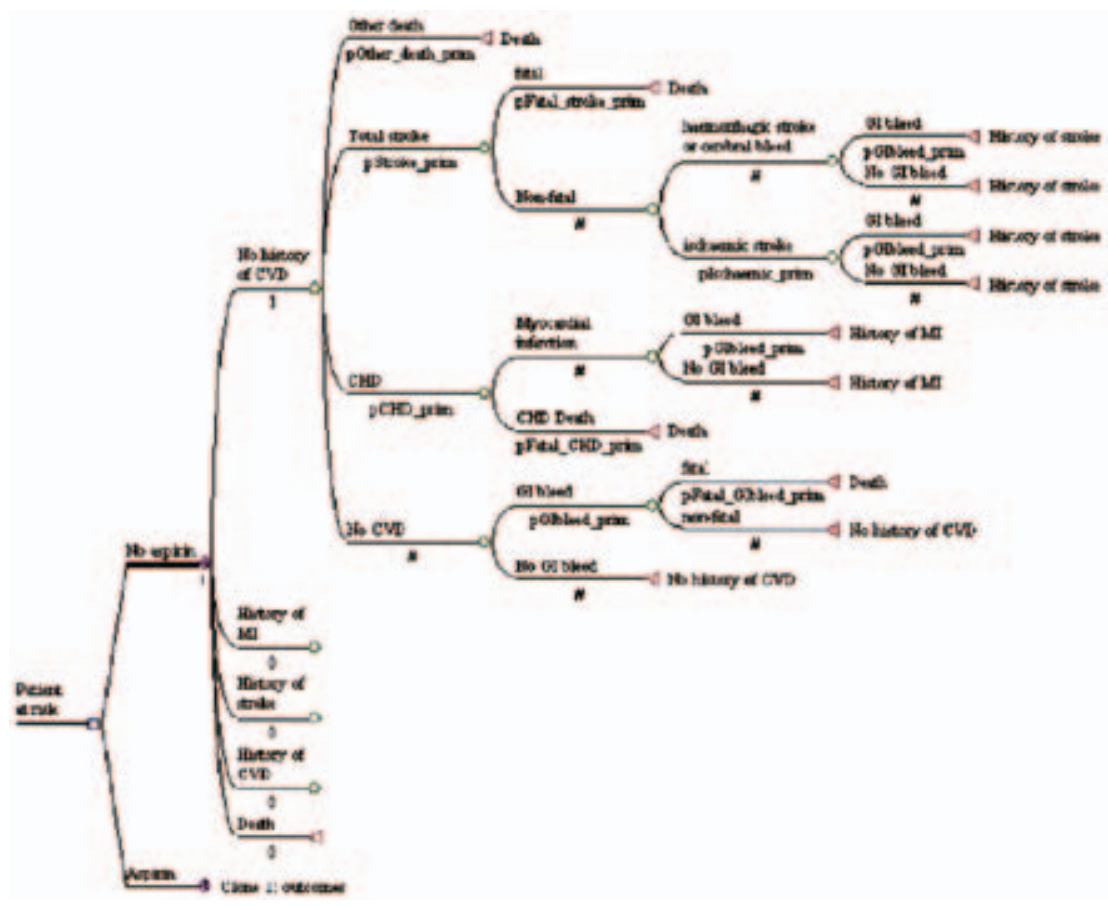

Figure 2. Rough sketch of Markov model used in the analysis. GI: gastro-intestinal, CHD: coronary heart disease, CVD: cardiovascular disease, MI: myocardial infarction.

(10-year risk of $\pm 15 \%$ ) were selected, referring to guideline in Europe/North America $(6,7)$. Discounting was applied $3 \%$ on cost and effects.

\section{Clinical data}

For annual transition probability of primary prevention, the results of the meta-analyses performed on the 5 trials that studied aspirin in primary prevention of CHD (Hayden et al 2002) (4) and Eidelman et al (2003) (5) were used.

The risk of events with aspirin and placebo is shown in Table 1, assuming an annual risk of CHD of $1.5 \%$.

With regard to the transition probability of secondary prevention, the results of the aspirin group in the CAPRIE (1996) trial (2) were used. The annual event rates for the aspirin treated group are reported in Table 2. If patients had a history of both events the maximum risk of stroke and MI was used.

\section{Economic data}

Direct costs were estimated from the insurers' perspective, and cost data used in this model are shown in Table 3. While international guidelines state that doses of $75 \mathrm{mg}$ and $325 \mathrm{mg}$ aspirin are equally effective, the base case calculations were done based on the use of $100 \mathrm{mg}$ aspirin (JPY 6.4 per day) both in primary and secondary prevention.

\section{Cost-effectiveness}

The incremental cost effectiveness of each treatment arm during a 10-year time horizon with 1-year cycles was applied and compared.

\section{Sensitivity analysis}

The robustness of the results of cost-effectiveness analysis was estimated by sensitivity analysis.

\section{Results}

\section{Base case analysis}

Based on an annual risk of CHD of $1.5 \%$ aspirin is significantly cost saving as shown in Table 4. 10-year costs were JPY 634,000 (Euro 4,857) and JPY 518,000 (Euro 3,968 ) in 'No aspirin' arm and 'aspirin' arm, respectively, while the life-years were 8.33 and 8.36 , respectively. Lowdose aspirin treatment saved on average JPY 116,000 (Euro 889) [95\% confidence interval (CI) JPY 57,077-175,151] per patient in Japan. And since more life year (LY) was gained with low-dose aspirin for a lower cost, administering aspirin proved to be the dominant (best) treatment option.

\section{Sensitivity analysis}

\section{(1) Annual risk of CHD}

In the base case the annual risk of CHD was set at $1.5 \%$. Here the annual risk was varied between 0 and $1 \%$ in order to search for the breakeven between 'aspirin' and 'no aspirin' arm. The result indicated that from the point of an annual risk of $0.20 \%$, low-dose aspirin was dominant to placebo (Fig. 3). And, the difference of 10-year costs become larger as the annual risk of CHD increased.

(2) Cost of stroke treatment

According to a survey, the weighted mean cost of stroke treatment (Hospitalization) was calculated to be around JPY 
Table 1. Summary Table of Annual Risk of Events in Primary Prevention, Assuming that Annual Risk of CHD is $1.5 \%$

\begin{tabular}{lcc}
\hline Outcome & No aspirin & aspirin \\
\hline CHD & $\mathbf{1 . 5 0 \%}$ & $\mathbf{1 . 0 9 \%}$ \\
Fatal CHD & $(25.8 \%)$ & $(31.0 \%)$ \\
Non-fatal CHD & $(74.2 \%)$ & $(69.0 \%)$ \\
\hline Stroke & $\mathbf{0 . 8 1 \% *}$ & $\mathbf{0 . 8 2 \%}$ \\
Fatal stroke & $(13.4 \%)$ & $(1.02 * \mathrm{X} \mathrm{0.81 \% )}$ \\
Non-fatal stroke & $(86.6 \%)$ & $(17.2 \%)$ \\
Hemorrhagic stroke & $(14.3 \%)$ & $(82.8 \%)$ \\
Ischemic & $(85.7 \%)$ & $(16.7 \%)$ \\
\hline GI bleeding & $\mathbf{0 . 1 8 \%}$ & $\mathbf{0 . 3 1 \%}$ \\
\hline Other death & & $(1.70 * \mathrm{X} 0.18 \%)$ \\
\hline
\end{tabular}

* from Odds ratio of Hayden's report ${ }^{(4)}$

CHD: coronary heart disease, GI: gastro-intestinal.

Table 2. Annual Risk Rates Reported for the Aspirin Group in the CAPRIE Trial for Secondary Prevention (2)

\begin{tabular}{|c|c|c|c|}
\hline Event & History of MI & History of stroke & $\begin{array}{l}\text { History of CVD } \\
\text { (MI and stroke) }\end{array}$ \\
\hline Non fatal stroke & $0.58 \%$ & $5.39 \%$ & $5.39 \% *$ \\
\hline Non fatal MI & $2.60 \%$ & $0.62 \%$ & $2.60 \% *$ \\
\hline Intracranial hemorrhage & $0.26 \%$ & $0.26 \%$ & $0.26 \%$ \\
\hline Vascular death & $1.66 \%$ & $1.71 \%$ & $2.06 \%$ \\
\hline Other death & $1.05 \%$ & $1.05 \%$ & $1.05 \%$ \\
\hline Fatal and non-fatal GI & & & \\
\hline bleeds & $1.40 \%$ & $1.40 \%$ & $1.40 \%$ \\
\hline
\end{tabular}

*Max. of second and third column

CAPRIE: clopidogrel versus aspirin in patients at risk of ischemic events, CVD:

cardiovascular disease, GI: gastro-intestinal, MI: myocardial infarction.

800,000 (Euro 6,154) (9). However, this data was based on the cost for patients with a JCS score of less than 30. As the real cost including more severe patients with a JCS score equal to or greated than 30 would be higher than this, the base case cost of stroke treatment was set to be JPY 1 million (Euro 7,692). Then, in the sensitivity analysis, the cost of stroke treatment was varied between JPY 100,000 (Euro 769) and JPY 10 million (Euro 76,920) in order to search for the breakeven between 'aspirin' and 'no aspirin' arms. The results indicated that the 'aspirin' arm was dominant to 'no aspirin' arm, regardless of the cost of stroke treatment.

(3) Time horizon

In the base case the time horizon was set at 10 years. Here, the time horizon was varied between 1 and 20 years. The result indicated that dominance (non-significant) was demonstrated in the first year of treatment (Fig. 4).

(4) Other factors
Table 3. Cost of Cardiovascular Disease in Japan

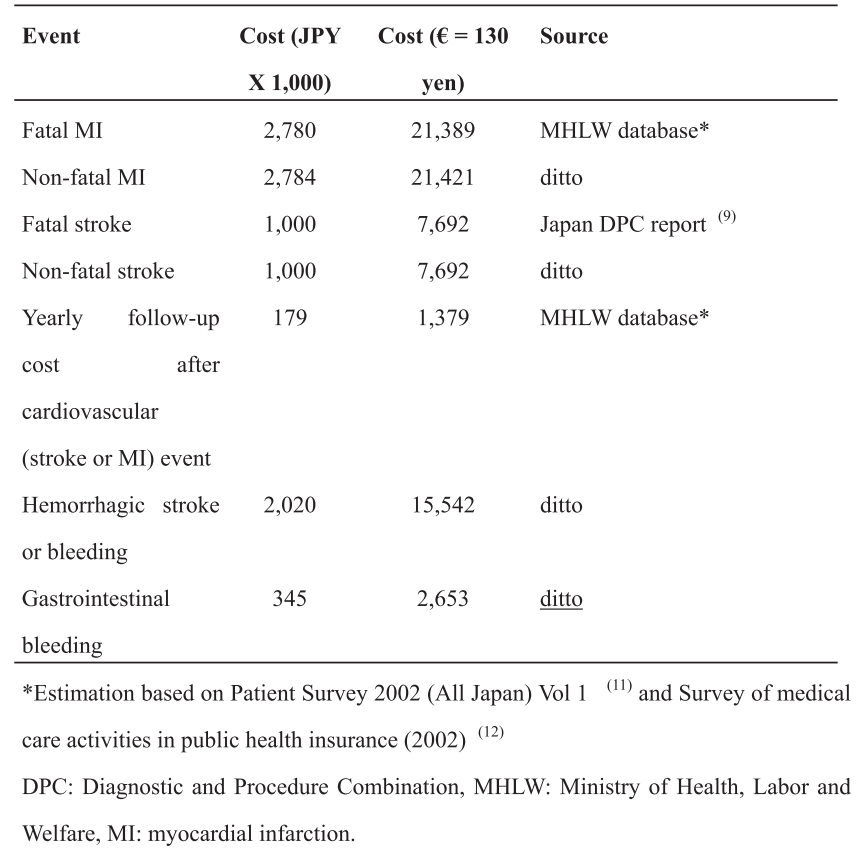

Table 4. Result of Markov Model

\begin{tabular}{lccccc}
\hline & Cost & Incr Cost & LY & LYG & Incr C/E ratio \\
\hline Japan & & & & & \\
No aspirin & 634,383 & & 8.33 & & \\
Aspirin & 518,269 & $-116,114$ & 8.36 & 0.03 & Dominant \\
\hline
\end{tabular}

LY: life year, LYG: life year gained, C/E: cost-effectivenes.

Sensitivity analysis on GI bleeding rate, stroke rate, cost of each event and discounting showed the robustness of the results.

\section{Discussion}

CVD causes serious side effects, such as speech disturbance and paralysis of extremities, and often requires longterm care. This not only impairs Quality of Life (QOL) of the patients, but also causes an increase in NHI budget. Hence, the importance of decreasing CVD in patients at high risk of CVD is now being stressed.

Recently, the effects of aspirin on the occurrence of CVD in patients at high CVD risk was reported by Hayden et al, based on meta-analysis of 5 clinical trials (4). In their report, the authors calculated that when the five-year absolute risk of coronary events reached $5 \%$ (annual risk of $\pm 1 \%$; 10 -year risk of $\pm 10 \%$ ), aspirin treatment reduced the absolute risk of coronary events by 0.3 percent per year but increased the risk of hemorrhagic stroke by 0.02 percent per year and the risk of major gastrointestinal bleeding by 0.06 percent per year.

A more clear presentation of the results of the metaanalysis is as follows: Per 1000 patients with a 5\% risk for 


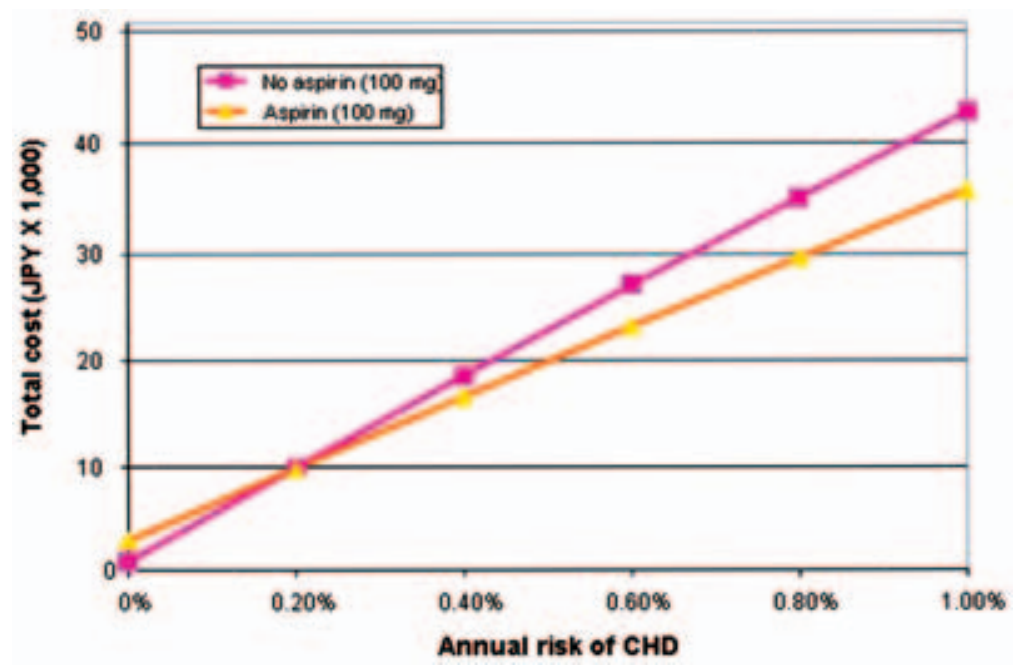

Figure 3. Sensitivity analysis of annual risk of CHD (10 years). CHD: coronary heart disease.

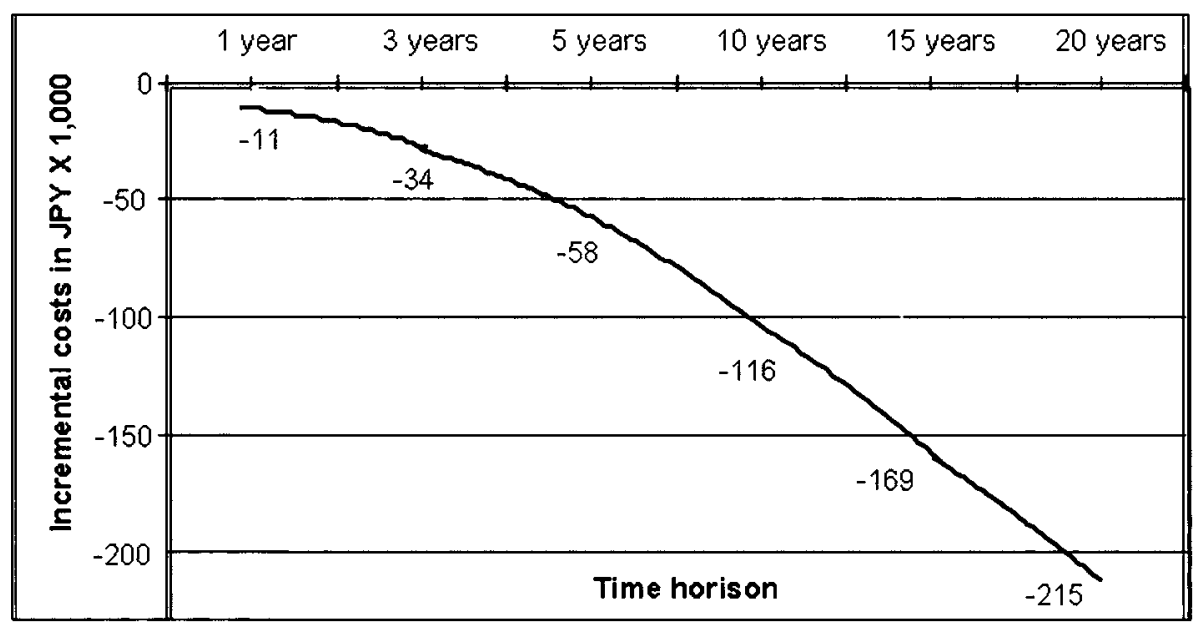

Figure 4. Sensitivity analysis of Markov cycle. Time Horizon (Annual risk of CHD = 1.5\%).

coronary heart disease (CHD) events (fatal and non-fatal) over 5 years, aspirin would prevent 6 to 20 myocardial infarctions. However, it would also cause 0 to 2 hemorrhagic strokes and 2 to 4 major gastrointestinal bleeding events. For patients with a CHD risk of $1 \%$ over 5 years, aspirin would prevent 1 to 4 myocardial infarctions but would still cause 0 to 2 hemorrhagic strokes and 2 to 4 major gastrointestinal bleeding events. Hence, Benefit/Risk of aspirin depends on the baseline risk of CHD in the studied population.

In response, the new European Guidelines (7) emphasize the importance of prevention in asymptomatic individuals who are at high risk of developing atherosclerotic CVD. The guidelines state that "there is evidence that low-dose aspirin can reduce the risk of cardiovascular events in people with diabetes, in people with well-controlled hypertension and in men at high multifactorial CVD risk". The American Heart Association also recommends starting treatment with aspirin as soon as the risk of a cardiac event is higher than $10 \%$ over a period of ten years (annual risk $\pm 1 \%$ ) (6).

The health economic analysis performed here showed that, from the perspective of the health care payer, in pa- tients with a moderately increased annual risk of CHD (1.5\%: 10-year risk $\pm 15 \%$ ), administering aspirin $100 \mathrm{mg}$ per day is cost saving (more effects are gained for a lower cost) in Japan, as well. The reliability of these results was demonstrated by the sensitivity analyses, and aspirin was demonstrated as being costeffective from an annual risk of $0.2 \%$.

This analysis supports the international recommendations for primary CVD prevention from both clinical and economic perspective. Furthermore, it is quite important from the regulatory perspective that savings were made as early as the first year of therapy. In addition, the result is robust through a different NHI system (8). However, it will be necessary to make various considerations before generalizing the results.

First, this study was based on the results of metaanalyses, which included studies with very different study periods, different baseline risks of CHD, and different doses of aspirin (ranging from $75 \mathrm{mg} /$ day to $500 \mathrm{mg} /$ day). Also, non-published details on the meta-analyses and single studies were not included in the analysis.

Second, costs of co-administration, such as antacid, were 
not included in the analysis. In clinical practice, antacid is sometimes co-administered with aspirin for decreased incidence of GI bleeding, while the price of antacid varies considerably by product (eg, ranging from magnesium oxide with JPY 0.3-3 per day, to omeprazol with JPY 134.1 per tablet), and while there would also be a possibility that antacid reduces the risk and cost of GI bleeding.

Third, the current estimation was based on foreign clinical data, and Japanese data was not included. Although the model targeted patients with increased CVD risk, benefit/ risk under aspirin treatment might not be comparable between Japanese and Caucasians. In addition, the difference of benefit/risk in gender should also be considered as well as ethnic difference, as a recent publication demonstrated that aspirin lowered the risk of stroke among women without affecting the risk of myocardial infarction (10).
Taking these points into account, further analyses based on clinical evidence of primary prevention and clinical practice in Japan are necessary to reconfirm the current results. Currently, some large-sized clinical research trials are ongoing in Japan (Japanese Primary Prevention Project (JPPP): http://www.clinicaltrials.gov/ct/show/NCT00110448?order=1, Japases Primary Prevention of Atherosclerosis with Aspirin for Diabetes (JPAD): http://www.clinicaltrials.gov/ct/show/ NCT00225849?order=1), and the results of these studies are awaited.

In conclusion, based on the results of this health economic evaluation, aspirin therapy should be recommended in the primary prevention of CVD in all individuals who have at least a moderately increased risk of CHD and who do not have an increased risk of GI bleeding events.

\section{References}

1. Kokumin-Iryohi-no-Gaikyo (National Medical Care Expenditure 2003 ) http://www.mhlw.go.jp/toukei/saikin/hw/k-iryohi/03/index. html. (in Japanese).

2. CAPRIE Steering Committee. A randomised, blinded, trial of clopidogrel versus aspirin in patients at risk of ischaemic events (CAPRIE). Lancet 348: 1329-1339, 1996.

3. Antithrombotic Trialists' Collaboration. Collaborative metaanalysis of randomised trials of antiplatelet therapy for prevention of death, myocardial infarction, and stroke in high risk patients. BMJ 324: 71-86, 2002 (Erratum in: BMJ 324: 141, 2002).

4. Hayden M, Pignone M, Phillips C, Mulrow C. Aspirin for the primary prevention of cardiovascular events:A summary of the evidence for the U.S. Preventive Services Task Force. Ann Intern Med 136: 161-172, 2002.

5. Eidelman RS, Herbert PR, Weisman SM, Hennekens CH. An update on aspirin in the primary prevention of cardiovascular disease. Arch Intern Med 163: 2006-2010, 2003.

6. Pearson TA, Blair SN, Daniels SR, et al. AHA Guidelines for Primary Prevention of Cardiovascular Disease and Stroke:2002 Update:Consensus Panel Guide to Comprehensive Risk Reduction for Adult Patients Without Coronary or Other Atherosclerotic Vascular
Diseases. American Heart Association Science Advisory and Coordinating Committee. Circulation 106 (3): 388-391, 2002.

7. De Backer G, Ambrosioni E, Borch-Johnsen K, et al. European guidelines on cardiovascular disease prevention in clinical practice. Third Joint Task Force of European and Other Societies on Cardiovascular Disease Prevention in Clinical Practice. Eur Heart J 24: 1601-1610, 2003.

8. Lamotte M, Annemans L, Evers T, et al. A multi-country economic evaluation of low-dose aspirin in the primary prevention of cardiovascular disease. Pharmacoeconomics 24: 155-169, 2006.

9. Ishikawa BK, Matsuda S. Kyu-sei-ki Nyu-in Iryo Shiko Shin-dan Bunrui (Survey using Diagnostic and Procedure Combination). Number H13-SEISAKU-034, Supple 3-1, 2004 (in Japanese).

10. Ridker PM, Cook NR, Lee IM, et al. A randomized trial of lowdose aspirin in the pimary prevention of cardiovascular disease in women. N Engl Med J 352: 1293-1304, 2005.

11. Kanjya-Chosa 2002 (Patient Survey 2002). http://www.mhlw.go. $\mathrm{jp} /$ toukei/itiran/gaiyo/k-eisei.html (in Japanese).

12. Shakai-Iryo Shinryo-koi-betsu Chosa 2002 (Survey of National Medical Care Insurance Service 2002). http://www.mhlw.go.jp/ toukei/saikin/hw/sinryo/tyosa02/index.html. (in Japanese).

(C) 2007 The Japanese Society of Internal Medicine http://www.naika.or.jp/imindex.html 